Copyright ( 2013 IEEE. Personal use of this material is permitted. Permission from IEEE must be obtained for all other uses, in any current or future media, including reprinting/republishing this material for advertising or promotional purposes, creating new collective works, for resale or redistribution to servers or lists, or reuse of any copyrighted component of this work in other works. 


\title{
New Insights Into Optimal Acoustic Feedback Cancellation
}

\author{
*C. Renato C. Nakagawa ${ }^{1}$, Student Member, IEEE, Sven Nordholm ${ }^{2}$, Senior Member, IEEE, Wei-Yong Yan ${ }^{3}$
}

\begin{abstract}
In this paper we present new insights into the bias problem for acoustic feedback cancellation when a probe signal approach is used. The optimum solution of the feedback canceler is not the feedback path but the product of the feedback path and the sensitivity function and hence, the solution is biased. The novelty of this paper also consists of the derivation of the conditions for unbiased feedback cancellation when a probe signal is used as input to the canceler. An adequate delay in the forward path is necessary to reduce, or remove the bias term. The theoretical analysis is verified with simulation results.
\end{abstract}

Index Terms-Acoustic feedback, bias problem, feedback cancellation, hearing aids, probe injection

\section{INTRODUCTION}

Acoustic feedback poses a problem in the normal operation of assistive listening devices due to the acoustic coupling between the loudspeaker and microphone. The microphone picks up the loudspeaker signal and re-amplifies it creating an acoustic loop, thus the signal traveling around this loop gets stronger for each round trip potentially causing stability problems. The feedback limits the maximum stable gain (MSG) achievable, it deteriorates the sound quality by producing a distortion of the incoming signal, and it is a cause of instability in acoustic systems working in closed-loop [1].

The use of feedback cancellation techniques is currently a preferred option to tackling the feedback problem [1]. The main challenge with traditional feedback cancelers is the well known bias problem. The biased solution in the canceler's estimate is caused by the correlation between the loudspeaker and incoming signal [1], [2]. It generally leads to a poor system performance and in the worst-case scenario, it causes the cancellation system to fail. Different techniques have been proposed to reduce this correlation including phase modification, frequency shifting, non-linear processing, decorrelating pre-filters, probe noise injection, and the use of multiple microphones to estimate the incoming signal and remove it prior to adapting the canceler [1], [3]-[6].

In this paper we study the adaptive feedback canceler's optimal solution when a probe signal is injected into the system. With a probe signal injection approach, either the probe or the loudspeaker signal can be used as an input to the adaptive canceler [3]. If the loudspeaker signal is used as an input to the canceler then the optimal solution results in the well known bias problem, where the correlation between

Department of Electrical and Computer Engineering, Curtin University, Kent Street, Bentley, WA 6102, Australia

1 carlos.nakagawa@postgrad.curtin.edu.au

2 s.nordholm@curtin.edu.au

${ }^{3}$ w.yan@exchange.curtin.edu.au

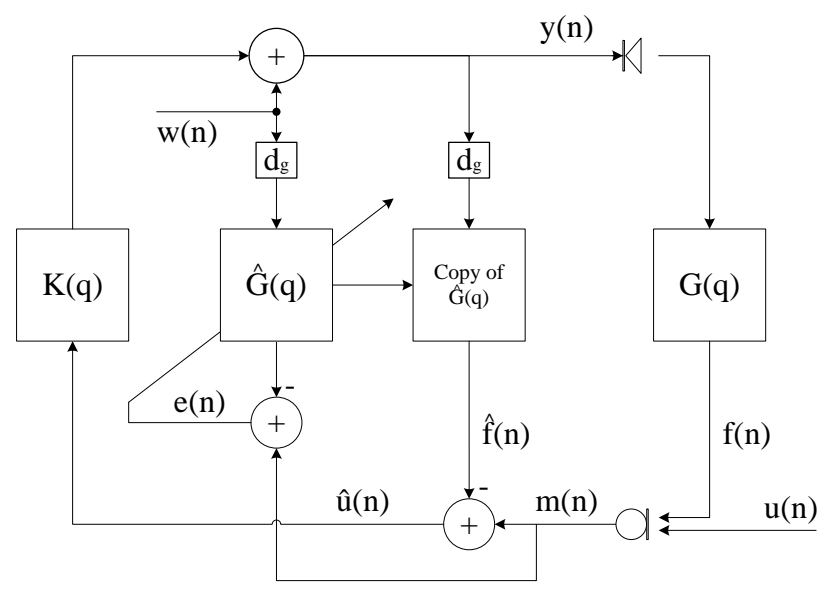

Figure 1. Injected probe signal approach in acoustic feedback cancellation.

the loudspeaker signal and incoming signal is of interest. However, if the probe signal, designed to be uncorrelated with the incoming signal, is used to drive the adaptive canceler, then it is accepted that the solution is an unbiased estimate of the feedback path see [3], [7] and references therein. However, we show theoretically that the injection of a probe signal, with the probe signal used as an input to the adaptive canceler, does not guarantee an unbiased solution. Thus, new insights into the bias problem is presented.

Section II presents the system description for acoustic feedback cancellation using a probe signal approach. Then, Section III establishes theoretical expressions for the optimum solution and presents new insights into the bias problem. Once the biased solution is recognized, conditions under which it can be reduced, or removed, is proposed. Finally, Section IV produces simulations results that verify the derived theoretical expressions.

In this paper, column vectors are emphasized using lower letters in bold. The superscript $\mathrm{T}$ denote vector transpose, the expectation operator is denoted by $E\{\cdot\}$, the discretetime index is denoted by $n$, and the symbol $q^{-1}$ denotes the discrete-time delay operator $q^{-1} u(n)=u(n-1)$. All signals are real-valued, and we denote all signals as discretetime signals with time index $n$ for convenience.

\section{SYSTEM DESCRIPTION}

Fig. 1 illustrates a feedback canceler for an assistive listening device with a single microphone. 
The feedback path between the loudspeaker and the microphone is assumed to be a discrete-time finite impulse response (FIR) filter with coefficient vector $\mathrm{g}=$ $\left[\begin{array}{llll}g_{0} & g_{1} & \cdots & g_{L_{g}-1}\end{array}\right]^{T}$ with filter length $L_{g}$ which is represented as a polynomial transfer function $G(q)$ in $q$ as $G(q)=\mathbf{g}^{T} \mathbf{q}$ with $\mathbf{q}=\left[\begin{array}{llll}1 & q^{-1} & \ldots & q^{-L_{g}+1}\end{array}\right]^{T}$. This representation allows the following notation, for the filtering of $y(n)$ by $G(q)$ [8],

$$
G(q) y(n)=\mathbf{g}^{T} \mathbf{y}(n) .
$$

Typically, the acoustic feedback path $G(q)$ contains a delay $d_{g}$ that arises from the processing delay of the analogueto-digital converter (ADC) and digital-to-analogue converter (DAC), i.e., $G(q)=q^{-d_{g}} \bar{G}(q)$ with $L_{g}=d_{g}+L_{\bar{g}}$ [9]. The feedback path $G(q)$ is therefore modeled as a cascade of a delay $d_{g}$ and a feedback canceler $\hat{G}(q)$. The adaptive filter $\hat{G}(q)$ identifies and tracks changes to the feedback path producing an estimate $\hat{f}(n)$ of the feedback signal $f(n)$. The loudspeaker and microphone signals are $y(n)$ and $m(n)$, respectively. The incoming signal is denoted by $u(n)$ and the feedback signal is denoted by $f(n)=G(q) y(n)$. The estimate $\hat{f}(n)$ is subtracted from the microphone signal $m(n)$.

A probe noise signal $w(n)$, that is designed to be uncorrelated to $u(n)$, is injected into the loudspeaker signal $y(n)$ and used as the input to the feedback canceler $\hat{G}(q)$.

The forward path $K(q)$ represents the regular signal processing path of the device (i.e., a frequency-specific gain, compression and/or noise reduction). In this paper, $K(q)$ has a delay $d_{k}$ of at least one sample and provides the system with a constant gain $\bar{K}(q)=\bar{K}$, i.e., $K(q)=q^{-d_{k}} \bar{K}(q)$.

A standard criterion to find an optimal set of coefficients is to minimize the mean square error (MSE) cost function

$$
J(\hat{\mathbf{g}})=E\left\{|e(n)|^{2}\right\} .
$$

From Fig. 1, it can be seen that

$$
m(n)=u(n)+G(q) y(n),
$$

and

$$
y(n)=K(q)(m(n)-\hat{G}(q) y(n))+w(n) .
$$

where we set $d_{g}=0$ to simplify equations but it does not impact the results. Nevertheless, the delay $d_{g}$ will be used in our simulations presented in Section IV.

Substituting (3) into (4)

$$
\begin{aligned}
y(n) & =S(q) K(q) u(n)+S(q) w(n) \\
& =S(q) \bar{y}(n)
\end{aligned}
$$

where $S(q)$ is the sensitivity function

$$
\begin{gathered}
S(q)=\frac{1}{1-K(q)(G(q)-\hat{G}(q))}, \\
\bar{y}(n)=K(q) u(n)+w(n),
\end{gathered}
$$

and

$$
e(n)=m(n)-\hat{G}(q) w(n)
$$

The frequency function

$$
K(\omega)(G(\omega)-\hat{G}(\omega))
$$

in (6) is often referred to as the "loop-response", where the spectrum of $K(q)$ and $G(q)$ is denoted by $K(\omega)$ and $G(\omega)$, respectively, with $\omega=[0,2 \pi]$. It plays a central part in acoustic feedback control [1], [3]. The Nyquist criterion states that oscillations may occur if the magnitude response of the loopgain is greater than unity and the loop-phase is a multiple of $2 \pi$ [10]. It can be seen in (6) that the channel $G(q)$ may lead to system instability. To avoid this, the amount of gain $K(q)$ has to be limited. However, if the feedback canceler $\hat{G}(q)$ can resemble $G(q)$, then the system is brought closer to its desired response $S(q)=1$. Ideally, $\hat{G}(q)=G(q)$ which results in $S(q)=1$.

\section{NEW INSIGHTS INTO THE BIAS PROBLEM USING PROBE SIGNAL INJECTION}

This section highlights the fact that there is some bias in the optimal solution when a probe signal is used to drive the feedback canceler. Furthermore, it presents conditions in which the solution's bias can be reduced or even removed completely.

Fig. 1 illustrates the case where $w(n)$ is the input to the feedback canceler. Minimizing the mean square error (MSE) cost function in (2), i.e.,

$$
\frac{\delta E\left\{|e(n)|^{2}\right\}}{\delta \hat{\mathbf{g}}^{T}}=0
$$

results in the Wiener filter [11]

$$
\hat{\mathrm{g}}_{o}=E\left\{\mathbf{w}(n) \mathbf{w}^{T}(n)\right\}^{-1} E\{\mathbf{w}(n) m(n)\}
$$

where

$$
\mathbf{w}(n)=\left[\begin{array}{llll}
w(n) & w(n-1) & \ldots & w\left(n-L_{\hat{g}}+1\right)
\end{array}\right]^{T}
$$

and $\hat{\mathrm{g}}_{o}$ is the set of optimal coefficients.

Using (5)-(7) we expand (3) as

$$
\begin{aligned}
m(n) & =u(n)+A_{F I R}(q) \bar{y}(n)+\left(A(q)-A_{F I R}(q)\right) \bar{y}(n) \\
& =u(n)+\bar{K} \cdot A_{F I R}(q) u\left(n-d_{k}\right)+A_{F I R}(q) w(n)+\xi(n)
\end{aligned}
$$

where

$$
A(q)=G(q) S(q)
$$

is a causal IIR filter, which may be specified as $A(q)=a_{0}+$ $q^{-1} a_{1}+\ldots$ The filter $A_{F I R}(q)$ corresponds to the first $L_{\hat{g}}$ coefficients of $A(q)$ and

$$
\begin{aligned}
\xi(n) & =\left(A(q)-A_{F I R}(q)\right) \bar{y}(n) \\
& =q^{-L_{\hat{g}}} A_{r}(q) \bar{y}(n)
\end{aligned}
$$

represents the residual impulse response $q^{-L_{\hat{g}}} A_{r}(q)$.

Assuming a sufficient-order filter and using (13) in (11) results in

$$
\begin{aligned}
\hat{\mathbf{g}}_{o} & =\mathbf{a}_{F I R}+E\left\{\mathbf{w}(n) \mathbf{w}^{T}(n)\right\}^{-1} E\{\mathbf{w}(n) \xi(n)\} \\
& =\mathbf{a}_{F I R}+E\left\{\mathbf{w}(n) \mathbf{w}^{T}(n)\right\}^{-1} E\left\{\mathbf{w}(n) \mathbf{w}_{a_{r}}^{T}\left(n-L_{\hat{g}}\right)\right\}
\end{aligned}
$$


as $w(n)$ and $u(n)$ are uncorrelated by construction of $w(n)$. In this paper we assume that $w(n)$ is a white Gaussian noise sequence and, as a result, $E\left\{\mathbf{w}(n) \mathbf{w}^{T}\left(n-L_{\hat{g}}\right)\right\}=\mathbf{0}$ and (16) becomes

$$
\hat{\mathrm{g}}_{o}=\mathbf{a}_{F I R} .
$$

However, if the probe noise signal $w(n)$ is masked/shaped to reduce its influence on sound quality, then $E\left\{\mathbf{w}(n) \mathbf{w}_{a_{r}}^{T}\left(n-L_{\hat{g}}\right)\right\}$ may not be zero and, as a result, will contribute to the bias term in (16).

Therefore, assuming that $w(n)$ is white Gaussian noise, it is shown that the optimal solution of the feedback canceler $\hat{G}(q)$ is not the feedback path $G(q)$ but the product of the feedback path $G(q)$ and the sensitivity function $S(q)$ and hence, the solution is biased.

\section{A. Conditions for identifiability}

Now, we present conditions for identifiability where the desired solution $\hat{G}(q)=G(q)$ can be obtained from (17).

If we write (14) as

$$
A(q)=G(q)+q^{-d_{k}} A(q) \bar{K}(q) E(q)
$$

where

$$
E(q)=(G(q)-\hat{G}(q))
$$

then, it can be seen from (18) that as a delay, $d_{k}$, is contained in $K(q) E(q)$ the first $d_{k}$ coefficients of $A(q)$ coincide with the impulse response of the feedback path $\left[\begin{array}{lll}g_{0} & \ldots & g_{d_{k}-1}\end{array}\right]$, i.e., the first $d_{k}$ coefficients will not be biased. If $d_{k} \geq L_{g}$ then $G(q)$ can be completely obtained from the first $d_{k}$ coefficients of $A(q)$, such as,

$$
A(q)=g_{0}+\ldots+q^{-L_{g}+1} g_{L_{g}-1}+\ldots+q^{-d_{k}+1} a_{d_{k}-1}+\ldots
$$

As we have influence over the design of the forward path, we can vary $d_{k}$ to reduce, or even remove, the bias term. Thus, by using an adequate delay, $d_{k} \geq L_{g}$, the solution is decoupled and an unbiased optimal solution for $G(q)$ can be obtained from using the first $L_{g}$ coefficients of $A(q)$, assuming sufficient filter order.

Lower gain values for $K(q)$ could also be used to reduce the bias term, however, this goes against assistive listening devices' main objective which is to provide its users with an amplified signal to compensate for their hearing impairment.

If we define

$$
\hat{A}(q)=\hat{G}(q) S(q)
$$

as the canceler, then $\hat{A}(q)$ is an unbiased estimate of $A(q)$. It is interesting to consider what happens to the biased solution in (18) as $\hat{A}(q) \rightarrow A(q)$. From multiplying both sides of (19) by $S(q)$ we can write $E(q)$ as

$$
E(q)=\frac{A(q)-\hat{A}(q)}{1+K(q)(A(q)-\hat{A}(q))}
$$

then as $\hat{A}(q) \rightarrow A(q)$ it can be seen from (22) that $E(q) \rightarrow 0$ and, as such, the bias term is reduced over time if the system converges, i.e. $A(q) \rightarrow G(q)$.

\section{Simulation Verification}

The goal of the simulations is to verify the derived theoretical expression in (18). To assess the performance of the algorithm, the misalignment between the true feedback path $G(q)$ and $A(q)$ is used. The misalignment curve is defined, in the frequency domain, as

$$
\Delta(G(q), A(q))=10 \log _{10} \frac{\int_{0}^{\pi}|G(\omega)-A(\omega)|^{2} d \omega}{\int_{0}^{\pi}|G(\omega)|^{2} d \omega} .
$$

In order to perform simulations, experiments were first conducted to obtain the feedback path's characteristics and variations. The assistive listening device used in our experiments was a Sensear ear plug SP1x with $16 \mathrm{kHz}$ sampling rate with a modified firmware to suit our real time experiment requirements. Measurements were conducted in an anechoic chamber on a Brį̈œel \& $\mathrm{Kj} \ddot{i} \iota œ r$ head and torso simulator type $4128 \mathrm{C}$. The device's microphone was set to record while a Gaussian white noise probe signal $w(n)$ was being injected into the loudspeaker to excite the feedback path. With such recordings we were able to identify the path $G(q)$. Included in the feedback path are the characteristics of the loudspeaker, the microphone, the ADC, the DAC.

To reduce complexity, the feedback path is therefore modeled as a cascade of a delay $d_{g}$ and a shorter feedback canceler. The delay $d_{g}$ was set to 32 samples, $L_{g}=96$, and $L_{\hat{g}}=48$. The last 16 samples of $G(q)$ is not modeled as the main impulse is contained within the first 80 samples, see Fig. 2 for the feedback path characteristics. The incoming signal $u(n)=0$.

The update of the feedback canceler's coefficients, $\hat{g}$, with step size $\mu=0.01$ is performed using the normalized leastmean-square (NLMS) algorithm

$$
\hat{\mathbf{g}}(n)=\hat{\mathbf{g}}(n-1)+\frac{\mu}{\mathbf{w}(n)^{T} \mathbf{w}(n)} \mathbf{w}(n) e(n) .
$$

It can be seen from (18) that $K(q)=q^{-d_{k}} \bar{K}(q)$ has an influence on the amount of bias in the solution. It is expected that the higher the gain $\bar{K}(q)$ the more the solution will be biased. Also, the longer the delay $d_{k}$ in $K(q)$, the less the solution will be biased. With this in mind, we present two plots. In the first plot, the delay is fixed to its lowest value $d_{k}=1$ and the gain is varied. In the second plot, the gain $\bar{K}(q)$ is fixed and the delay $d_{k}$ varied. Each misalignment curve presented is an average of 50 simulations run where in each run a new realization of white Gaussian noise sequence is drawn for $w(n)$.

Fig. (3) presents the first plot where the gain is varied from $0 \mathrm{~dB}$ to $30 \mathrm{~dB}$ in $10 \mathrm{~dB}$ increments. The delay $d_{k}$ is kept constant $d_{k}=1$. As the gain is increased, the misalignment between $G(q)$ and $A(q)$ increases. If $K(q)$ were to be an open circuit, $A(q)=G(q)$ as per (18).

Fig. (4) presents the second plot where the delay $d_{k}$ is varied. Here the gain is kept constant $\bar{K}(q)=30 \mathrm{~dB}$. As the delay is increased, the misalignment curves shifts downwards as $K(q) E(q)$ and $G(q)$ are decoupled. If $d_{k} \geq L_{g}$ the misalignment value is $-\infty$, which is not shown in the plot. 

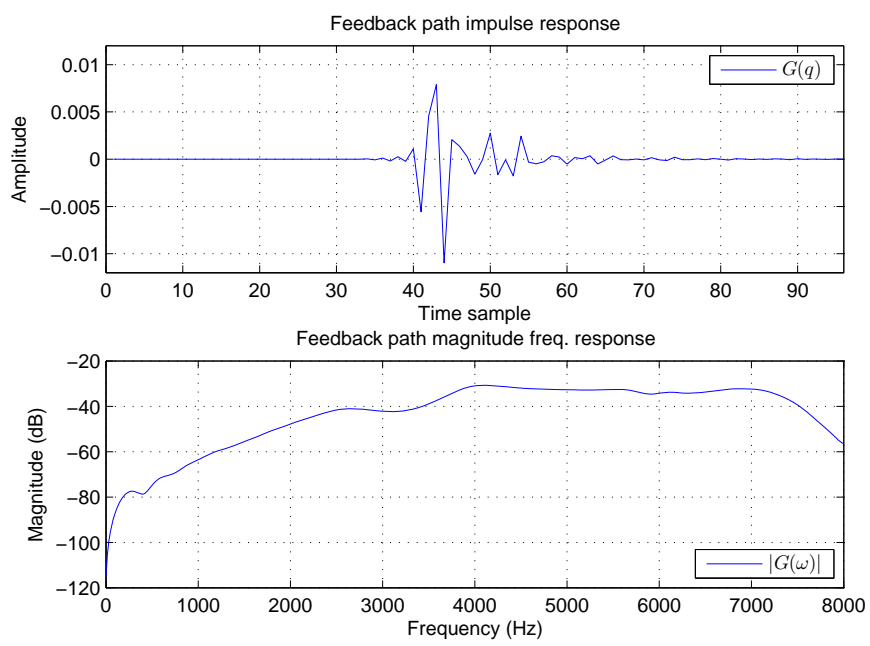

Figure 2. Feedback path characteristics.

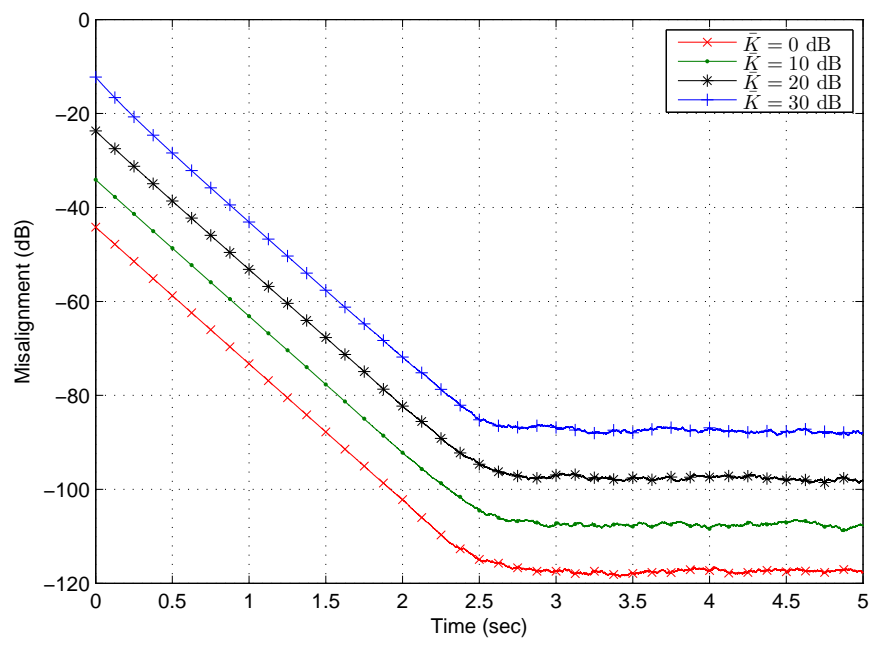

Figure 3. Misalignment between $G(q)$ and $A(q)$ with varying gain $\bar{K}$ with $d_{k}=1$. As the gain is increased, the misalignment between $G(q)$ and $A(q)$ increases. If $K(q)$ were to be an open circuit, $A(q)=G(q)$.

With both plots, it can be seen that the misalignment between $G(q)$ and $A(q)$ is reduced over time as $E(q) \rightarrow 0$ resulting in $A(q) \rightarrow G(q)$.

\section{CONClusion}

This paper presented new insights into the bias problem for acoustic feedback cancellation when a probe signal is used. It was presented, using theoretical results, that the feedback canceler's optimum solution is not the feedback path $G(q)$ but the product of the feedback path $G(q)$ and the sensitivity function $S(q)$ and hence, the solution is biased.

The novelty of this paper also consists of the derivation of the conditions for unbiased feedback cancellation when a probe signal is used as input to the canceler. It was demonstrated that by manipulating the forward path $K(q)$ the bias term resulting from the sensitivity function $S(q)$ can be reduced, and even removed. Lower gains and/or higher delays in the forward path results in a reduction of the solution's bias.

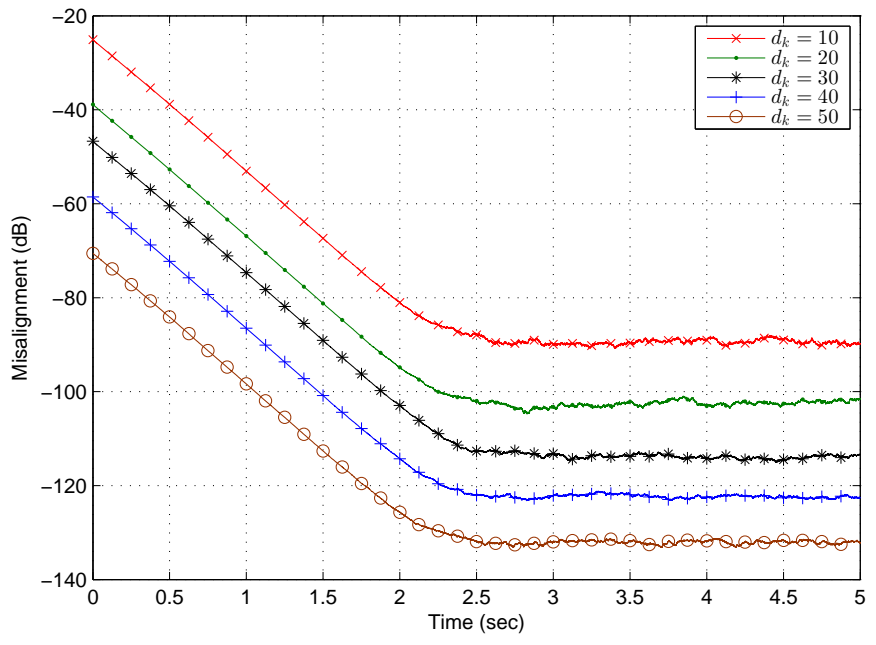

Figure 4. Misalignment between $G(q)$ and $A(q)$ with varying delay $d_{k}$ with $\bar{K}=30 \mathrm{~dB}$. As the delay is increased, the misalignment curves shifts downwards as $K(q) E(q)$ and $G(q)$ are decoupled. If $d_{k} \geq L_{g}$ the misalignment value is $-\infty$ (not shown in the plot). Also note that $d_{g}=32$. Plot with $d_{k}=1$ is presented in Fig. (3).

However, assistive listening devices normally require higher gains, so it is recommended to add an adequate delay, ideally $d_{k} \geq L_{g}$, to deal with the biased solution. Thus, by adding an adequate delay in the forward path an unbiased solution can be obtained.

The theoretical analysis was verified with simulation results.

\section{REFERENCES}

[1] A. Spriet, S. Doclo, and M. Moonen, "Feedback control in hearing aids." Springer handbook of speech processing, 2008.

[2] A. Spriet, I. Proudler, M. Moonen, and J. Wouters, "Adaptive feedback cancellation in hearing aids with linear prediction of the desired signal," IEEE Trans. Signal Process., vol. 53, no. 10, pp. 3749-3763, 2005.

[3] T. van Waterschoot and M. Moonen, "Fifty years of acoustic feedback control: state of the art and future challenges," Proceedings of the IEEE, no. 99, pp. 1-40, 2011.

[4] J. Hellgren and F. Urban, "Bias of feedback cancellation algorithms in hearing aids based on direct closed loop identification," IEEE Trans. Speech Audio Process., vol. 9, no. 8, pp. 906-913, 2001.

[5] C. R. Nakagawa, S. Nordholm, and W.-Y. Yan, "Dual Microphone Solution for Acoustic Feedback Cancellation for Assistive Listening," in ICASSP, Mar. 2012, pp. 149-152.

[6] J. Hellgren, "Analysis of feedback cancellation in hearing aids with Filtered-x LMS and the direct method of closed loop identification," IEEE Trans. Speech Audio Process., vol. 10, no. 2, pp. 119-131, 2002.

[7] M. Guo, S. H. Jensen, and J. Jensen, "Novel Acoustic Feedback Cancellation Approaches in Hearing Aid Applications Using Probe Noise and Probe Noise Enhancement," IEEE Trans. Audio, Speech and Language Process., vol. 20, no. 9, pp. 2549-2563, 2012.

[8] L. Ljung, System identification: theory for the user, 2nd ed. PrenticeHall, Aug. 1999.

[9] A. Spriet, G. Rombouts, and M. Moonen, "Adaptive feedback cancellation in hearing aids," J. Franklin Institute, 2006.

[10] H. Nyquist, "Regeneration Theory," Bell Telephone System, 1932.

[11] U. Forssell and L. Ljung, "Closed-loop identification revisited," Automatica, vol. 35, no. 7, pp. 1215-1241, Jul. 1999. 